\title{
Editorial
}

\section{Desafíos de la investigación en educación Matemática}

\author{
Alfonso Jiménez Espinosa ${ }^{1}$ (1) \\ Universidad Pedagógica y Tecnológica de Colombia \\ Editor Invitado
}

En esta época de cambio tan acelerado, en la cual la velocidad de producción de conocimiento es tal, que es imposible dar cuenta siquiera de cualquier reducida área de conocimiento, incluso para cualquier especialista, la investigación y la reflexión permanentes sobre la mejor forma de entender la complejidad del aula de clase, de la escuela y de la sociedad son necesidades absolutas. La investigación en educación matemática enfrenta serios desafíos para entender la poderosa influencia de la matemática en una sociedad tecnologizada y en una cultura dependiente de esta (Borba \& Skovsmose, 1997, 2001), para que la investigación no continúe haciendo más de lo mismo.

Para D'Ambrosio (1994), la tremenda influencia de la matemática en la cultura de la tecnologización de la sociedad ha hecho que la humanidad esté inmersa en una tecnonaturaleza; de tal forma, la tecnología se volvió un cuchillo de dos filos, ya que es una importante fuente de soluciones, pero también una causa de nuevos problemas. Es decir, la paradoja de la tecnología se ha convertido en un desafío para la investigación en educación matemática, pues cada vez más hay un gran sector de la sociedad que de alguna forma se ve afectada y marginada.

La matemática ha estado rodeada históricamente de cierto halo falso de súper-ciencia por la característica de racionalidad que engendra, que fue exaltada por Kant, al colocarla como el ejemplo por antonomasia del conocimiento a priori fruto de la razón humana, lo cual no ha sido aislado del poder que ejerce sobre otras áreas y el temor que ha generado 
en la cultura de la escuela, con su consiguiente poder de exclusión, generalmente de las clases menos favorecidas. Este poder se vuelve casi ilimitado con sus aplicaciones y más con la aparición de las TIC, donde "las matemáticas y la tecnología de la información no solo proporcionan descripciones y explicaciones de la realidad existente, [sino que] también crean una nueva realidad" (Keitel, 2004, p. 19). La matematización de todos los hechos y la modelización de los fenómenos de la naturaleza y de la vida, en palabras de Keitel, ha hecho que se consideren como formas y "ritos culturales inconscientes" (p. 20) y una forma de lenguaje que crea coacciones incuestionables y restricciones de la conciencia.

Las falsas creencias populares sobre las matemáticas y su refuerzo desde el mundo académico - preponderantemente racionalista, formalista y estructuralista- llevaron a que la práctica del profesor fuera un monólogo en el intento de "trasmitir" un mensaje conceptos matemáticos acabados, infalibles, sin sentido y sin contexto, ¡muertos! - a los niños y jóvenes (Jiménez, 2010). Pensando en una situación diferente, se debe promover una educación matemática como producto de la cultura Bishop $(1988,2005)$, en la cual la comunicación en clase debe tener otro enfoque, el de la búsqueda de una construcción colectiva a través de consensos para identificar, nombrar y nominar los conceptos, desde la participación y la argumentación de toda la clase (Jiménez-Espinosa, 2019; Jiménez, Suárez \& Galindo, 2010). No obstante, es claro que la educación matemática no puede quedarse ahí. Debe involucrarse en lo que Skovsmose (2001) llama "el direccionamiento a problemas", lo cual "implica que la dimensión del envolvimiento crítico debe hacer parte de la educación" (p. 20).

Lo anterior muestra la trascendencia de la investigación en educación matemática, la cual debe entender esta problemática y dar un giro a los estudios hacia la transformación social, donde los problemas de análisis no sean solo los de mejorar los aprendizajes, o los de mostrar competencias en pruebas censales, sino también el cuestionamiento de las verdades y paradigmas establecidos que generan tanta desigualdad e injusticia en todos los ámbitos. Esta tarea es un desafío para los investigadores en educación matemática, cuya divulgación estamos prestos a apoyar desde Praxis \& Saber con la edición de un nuevo dosier sobre educación matemática crítica.

En este número especial de educación matemática, se presentan trece textos que abarcan diversos enfoques, modalidades y temáticas. El primer bloque está compuesto por nueve artículos de investigación. Comienza con tres textos, fundamentados en la teoría del enfoque ontosemiótico [EOS] y más específicamente en los criterios de idoneidad didáctica del profesor de matemáticas. El primer artículo de Hummes, Breda, Seckel y Font muestra un estudio en un grupo de profesores y analiza sus prácticas, basados en las lesson study. Los criterios de idoneidad permiten ver que los profesores utilizan más los criterios interaccional y cognitivo; un poco menos los criterios epistémico y emocional; y los criterios de medios y ecológico son poco utilizados en la reflexión del grupo.

El segundo artículo, de Suárez y Suárez, analiza los criterios de idoneidad didáctica en un estudio de caso de un profesor de cálculo integral en grupos de ingeniería. Entre los resultados, se destaca que los procesos del profesor son los de institucionalización, representación, materialización, idealización y reificación. En algunas ocasiones se presentan procesos de significación y de generalización; mientras que por parte de los alumnos los 
procesos más comunes son los de representación, materialización e idealización. Muy pocas veces se presentan procesos de personalización y reificación, debido a que el docente no permite identificar en todos los estudiantes cuándo se hacen partícipes en la construcción de los significados.

El tercer texto, de Caicedo y Chacón, reporta una investigación que diseñó y validó un modelo didáctico para el aprendizaje de ecuaciones diferenciales, con énfasis en métodos cualitativos, basados en la concepción cuasiempírica de las matemáticas. Utilizan una metodología cualitativa, con estudiantes de ingeniería pertenecientes a un curso de ecuaciones diferenciales. Los resultados evidencian que los estudiantes se sienten motivados e interesados y que la metodología aplicada — basada en la resolución de problemas, con el uso de conjeturas desde la construcción del modelo hasta llegar a la solución final y el uso de la tecnología para estudiar los métodos cualitativos y numéricos- favorece la comprensión de los contenidos de estudio.

El cuarto artículo, de Pérez, Jaramillo y Ferreira, muestra el proceso de organización de enseñanza de tres profesores de matemáticas en una institución universitaria en un programa de administración, a partir de la creación colectiva de estudios de caso, con base en el método dialéctico para comprender el movimiento de este proceso de enseñanza. A partir del trabajo colectivo, los profesores crearon un caso sobre oferta y demanda, y expusieron un conocimiento matemático para aportar al análisis y solución de problemas empresariales. Se fundamentan en la teoría histórico-cultural, para la organización de la enseñanza como objeto de la actividad del profesor y los estudios de caso como metodología de enseñanza. Narran el proceso de creación del caso por parte de los profesores protagonistas y analizan el proceso de escritura que estuvo permeado por una intencionalidad de crearlo a partir de sus propias necesidades, experiencias y saberes, como una forma propia para mostrar el conocimiento matemático.

A continuación, se presentan dos artículos de investigación que usan el programa informático Geogebra. El primero de estos, de Joya y Suárez, presenta resultados de una investigación acerca del aprendizaje de los elementos notables del triángulo en geometría euclidiana, realizada con estudiantes de un grado décimo de educación media. Analizan la conjeturación y caracterización de dichos elementos a partir de la exploración de ambientes de geometría dinámica diseñados en GeoGebra. Adoptaron una metodología cualitativa de tipo descriptiva-interpretativa. La propuesta se basó en el aprendizaje por descubrimiento de Bruner. Hubo un progreso en el pensamiento intuitivo de los estudiantes al implementar las actividades centradas en la visualización y mediadas con dibujos dinámicos. Encuentran que los estudiantes aprendieron sobre rectas y puntos notables del triángulo al identificarlos en la representación gráfica, priorizar el uso del lenguaje natural, conjeturar sobre sus propiedades y descubrir relaciones y propiedades de los triángulos, mientras avanzan en el desarrollo del pensamiento espacial. Un elemento importante fue el desarrollo de la competencia digital de los estudiantes con el uso de GeoGebra.

El siguiente texto, de Ballesteros, Lozano y Rodríguez, reporta una investigación con el uso de la calculadora gráfica de GeoGebra. Presenta resultados del trabajo realizado en un curso de ingeniería. Se describen y se analizan los efectos de implementar una unidad didáctica para la enseñanza de la noción de área bajo la curva, durante un curso de 
cálculo integral, a partir de la integración de teléfonos inteligentes durante la experiencia de aprendizaje. Realizaron un diseño experimental de cuatro grupos de Solomon, con el propósito de determinar la influencia de una intervención mediada por la aplicación móvil Calculadora Gráfica de GeoGebra, y de verificar los efectos de una prueba de entrada sobre la cantidad de respuestas correctas en la prueba de salida. Los resultados fueron analizados mediante la aplicación de una prueba ANOVA de dos vías. Se encontró que el pretest tuvo una influencia directa en los resultados del postest y que los dos grupos que recibieron la intervención mediada por la aplicación móvil de GeoGebra obtuvieron mejor rendimiento en la prueba de salida que aquellos grupos que tuvieron una intervención con calculadora científica tradicional.

El octavo artículo, de Santiago, Vergel y Gallardo, reporta una investigación que tuvo como objetivo evaluar la relación entre el rendimiento académico en matemáticas y la variable éxito escolar en estudiantes de educación superior. La investigación siguió un enfoque cuantitativo, descriptivo-comparativo, de fuente de datos primaria, transeccional en la recogida de información, y microsociológico, en una cobertura muestral de 1500 estudiantes. Utilizaron la escala de resiliencia de Wagnild y Young. Los resultados reflejan que existe una relación significativa entre el nivel de exigencia, la aplicación, la abstracción y el rendimiento académico. Los estudiantes desarrollan habilidades de resiliencia emocionales que les permiten mejorar su calidad de vida. Concluyen que la resiliencia se relaciona significativamente con todas las fortalezas de carácter, así como con la aceptación positiva al cambio y la competencia personal. Identifican que, si bien la resiliencia ayuda a los estudiantes a ser más tolerantes y a comprender mejor lo que sucede en el entorno, es precisamente en esos desafíos donde se manifiestan las fortalezas que les brindan un alto desempeño educativo.

El último artículo de investigación, de Leguizamón, Jiménez y Chaparro, presenta un análisis sobre el quehacer docente de profesores de licenciatura en matemáticas, que consideran como un punto de partida para mejorar las prácticas de aula. Toman como referente teórico los modelos de clase propuestos por Porlán, complementados con el constructivismo clásico de Piaget, el enfoque sociocultural de Vygotsky y el interaccionismo de Bruner. Es una investigación de tipo mixto, con alcance descriptivo. Los principales instrumentos de recolección de información fueron cuestionarios y entrevista no estructurada. Presentan como resultados que, en general, los docentes que participaron diseñan sus clases según objetivos operacionales; evalúan mediante la aplicación de pruebas objetivas planteadas especialmente a manera de guías; y consideran que el aprendizaje ocurre mediante un proceso progresivo de asimilación de conceptos. De acuerdo con lo anterior, se evidencia que las características de las prácticas de los profesores se asocian con una tendencia didáctica tecnológica.

A continuación, se presentan dos artículos de reflexión. El primero, de Oliveira, analiza la Base Nacional Común Curricular brasileña [BNCC], la cual presenta marcados componentes políticos y es objeto de fuertes conflictos y tensiones en ese contexto educativo. El documento presenta implícitos los fundamentos formulados por Ball, tomados como base de los paradigmas que orientan esa construcción y las consecuencias ideológicas resultantes de la intervención de organismos multilaterales externos. Se hace un análisis de esas posiciones a través de cartas públicas y de entrevistas con un representante de la 
Sociedad Brasileña de Educación Matemática, en diferentes momentos del proceso de construcción del documento prescriptivo para el aprendizaje de la matemática en Brasil. Los análisis evidencian la necesidad de reflexiones sobre esos modelos verticales, que silencian voces y asumen los contenidos matemáticos como instrumentos de gestión con una determinación previa del proceso educativo y de las personas que se forman, que están implícitos en esos movimientos de reforma.

El siguiente artículo de reflexión, de Castellanos, busca incentivar a los educadores matemáticos para que razonen sobre el rol social, político y cultural del quehacer cotidiano en el aula de clase. El autor resalta la connotación política de la enseñanza de las matemáticas y la perspectiva sociopolítica como otra forma de orientar y compartir saberes. Abre una discusión al respecto de la connotación política de la matemática, la cual genera un panorama de monocultura del saber que ha permeado la enseñanza en la escuela regular y ha convertido el escenario escolar en un reproductor de la cultura hegemónica que determina qué conocimientos enseñar y cuáles no. Examinan la importancia de realizar una enseñanza de las matemáticas desde la pedagogía crítica. Se comprende que toda acción en el aula es política y tiene incidencia social. Al final explora algunas estrategias pedagógicas encaminadas a aproximar a educandos y educadores al saber matemático desde una práctica reflexiva y contextual, como estrategia para hacer una matemática cultural.

Más adelante, se presentan dos artículos de revisión. El primero, de Villamil y Riscanevo, analiza la construcción histórica y epistemológica del número cero, la cual sirvió como fundamento teórico de una investigación en el campo de la educación matemática, enfocada en categorizar las interpretaciones de estudiantes en formación inicial de matemáticas sobre el cero como número. El análisis permitió identificar aportes culturales de diferentes civilizaciones, entre los que se destacan: la interpretación dada por los egipcios como valor de referencia, su valor posicional dado por los mesopotámicos, el uso como cifra y como número dado en la India, entre otros. Además identificaron que las dificultades en su comprensión han perdurado, sin importar el contexto, al punto que trascienden y permean su enseñanza. Establecieron y fundamentaron una base conceptual y el reconocimiento de la historia como valor pedagógico en la enseñanza de las matemáticas, y su relación con las creencias y percepciones de la matemática, en articulación con la formación de profesores como campo de investigación.

El artículo de Montero y Mahecha parte de la preocupación de los docentes de básica primaria en Colombia por el bajo desempeño de los estudiantes en el área de matemáticas, los resultados obtenidos en las pruebas de estado y las dificultades en los procesos de comprensión de los enunciados de los problemas matemáticos. Este texto comparte una propuesta metodológica para contribuir al mejoramiento de los niveles de comprensión y resolución de problemas matemáticos, desde el concepto de macroestructura textual, con la integración de las áreas de matemáticas y de lenguaje. Los resultados de la revisión hacen parte de una investigación cualitativa en el marco de la investigación acción. En los resultados hacen evidentes las dificultades a la hora de resolver problemas matemáticos y diseñan una propuesta metodológica que integra la reconstrucción de macroestructuras en el análisis de enunciados, y el principio de interdependencia entre matemáticas y lenguaje.

Por último, se presentan dos trabajos: el primero, de tres docentes investigadores de 
la Universidad Francisco de Paula Santander, muestra a través de un estudio cualitativodescriptivo la importancia del uso de Derive para facilitar el aprendizaje del cálculo diferencial. El otro trabajo desarrolla una investigación de profesores de enfermería de la UPTC que consiste en identificar prácticas pedagógicas de docentes de enfermería utilizando perspectivas cualitativo-fenomenológicas.

\section{Referencias}

Bishop, A. (1988). Mathematical Enculturation. Dordrecht/Boston/London: Kluwer Academic Publishers.

Bishop, A. (2005). Aproximación sociocultural a la educación matemática (Trad. P. Perry,). Cali: Universidad del Valle; Instituto de educación y Pedagogía.

Borba, M. \&. Skovsmose, O. (2001). A ideologia da certeza em educação matemática. Em Skovsmose, O. (2001) Educação Matemática Crítica: A Questão da Democracia (127160). Campinas, S.P.: Papirus Editora.

Borba, M., \& Skovsmose, O. (1997). The ideology of certainty. For the Learning of Mahematics, (17), 17-23.

D'Ambrosio, U. (1984). Socio-cultural bases for mathematics education: A rationale for ethno-science [Material inédito]. Campinas S.P.: Universidade Estadual de Campinas.

Jiménez, A. (2010). La naturaleza de la matemática, las concepciones y su influencia en el salón de clase. Educación y Ciencia, (13), 155-162.

Jiménez, A., Suárez, N., \& Galindo, S. (2010). La comunicación: eje dela clase de matemáticas. Praxis \& Saber, 1(1), 173-202.

Jiménez-Espinosa, A. (2019). La dinámica de la clase de matemáticas mediada por la comunicación. Revista de Investigación, Desarrollo e Innovación, 10(1), 121-134. https://10.19053/20278306.v10.n1.2019.10016

Keitel, C. (2004). ¿Para qué necesitan nuestros estudiantes las matemáticas? En J. Giménez, L. Santos, J. \& Ponte (Coords.), La actividad matemática en el aula (11-24). Barcelona: Graó.

Skovsmose, O. (2001). Educação Matemática Crítica: A Questão da Democracia. Campinas, S.P.: Papirus Editora. 